\title{
A LOW-FREQUENCY ASYMPTOTIC MODEL OF SEISMIC REFLECTION FROM A HIGH-PERMEABILITY LAYER
}

\author{
DMITRIY SILIN ${ }^{1}$ AND GENNADY GOLOSHUBIN ${ }^{2}$ \\ ${ }^{1}$ Lawrence Berkeley National Laboratory, Berkeley, CA \\ ${ }^{2}$ University of Houston, TX
}

\begin{abstract}
Analysis of compression wave propagation through a high-permeability layer in a homogeneous poroelastic medium predicts a peak of reflection in the low-frequency end of the spectrum. An explicit formula expresses the resonant frequency through the elastic moduli of the solid skeleton, the permeability of the reservoir rock, the fluid viscosity and compressibility, and the reservoir thickness. This result is obtained through a low-frequency asymptotic analysis of the Biot's model of poroelasticity. A new physical interpretation of some coefficients of the classical poroelasticity is a result of the derivation of the main equations from the Hooke's law, momentum and mass balance equations, and the Darcy's law. The velocity of wave propagation, the attenuation factor, and the wave number, are expressed in the form of power series with respect to a small dimensionless parameter. The latter is equal to the product of the kinematic reservoir fluid mobility, an imaginary unit, and the frequency of the signal. Retaining only the leading terms of the series leads to explicit and relatively simple expressions for the reflection and transmission coefficients for a planar wave crossing an interface between two permeable media, as well as wave reflection from a thin highly-permeable layer (a lens).

The practical implications of the theory developed here are seismic modeling, inversion, and attribute analysis.
\end{abstract}

\section{INTRODUCTION}

Field observation show anomalous seismic signal reflection from a thin fluidsaturated permeable layer in the low-frequency end of the spectrum. Moreover, a frequency-dependent analysis of the data identifies the most productive spots in the reservoir [12, 21-27]. The classical theory of elasticity associates anomalous reflection with the tuning effect, which takes place when the thickness of the layer is equal to onefourth of the wavelength [44]. This is not the case in the situations mentioned in the beginning, where the thickness of the reservoir is much smaller that a quarter of the wavelength. Therefore, an explanation of the observed phenomenon based exclusively on the classical elasticity is insufficient, and the interaction between the elastic wave and fluid flow has to be taken into account as well.

Key words and phrases. Hooke’s law, Darcy’s law, poroelasticity, low frequency, permeability, asymptotic analysis, seismic imaging. 
The theoretical foundations of seismic wave propagation in a fluid-saturated porous medium have been established in the pioneer works by Frenkel, Gassmann, Biot, and others [5, 6, 15, 18-20, 36]. A comprehensive overview of the Biot's theory is presented in [40]. Further extensions accounting for local heterogeneities including double-porosity or layered media have been developed in $[3,10,11,29,31,41,42]$. The reflection and transmission coefficients predicted by the Biot's theory for a wave crossing a planar interface have been calculated in $[17,28]$. The complexity of the Biot's solution makes the expressions for the reflection and transmission coefficients very cumbersome. It makes it difficult to see how and what rock and fluid properties affect the magnitudes of the reflection and transmission coefficients. Even for the first-arrival reflection from a single or multiple layers, an analytical study is practically unfeasible. Therefore, simplified asymptotic relationships, valid in the low-frequency range, can help to obtain relatively simple and practically useful expressions for the reflection and transmission coefficients at seismic frequencies.

In this work, we develop an asymptotic analysis of elastic wave propagation in a fluid-saturated porous medium. The small parameter used in this analysis is equal to the product of the reservoir fluid mobility and density, and the frequency of the signal, multiplied by an imaginary unit. The velocities of the slow and fast Biot's waves and the respective wave numbers, as well as the attenuation factors, are expressed as power series with respect to this small parameter. The coefficients of the series are real-valued functions of the properties of the reservoir rock and fluid, but do not depend on the reservoir fluid mobility or the frequency of the signal. The approximate expressions obtained by retaining only the leading terms produce relatively simple mathematical expressions, which are valid in the low-frequency end of the spectrum including the seismic frequency band $(10-100 \mathrm{~Hz})$. Using these expressions, we study the reflection and transmission coefficients for a planar wave crossing a permeable interface at a normal incident angle. We further obtain that the fast-wave reflection coefficient from a thin permeable layer (a lens), as a function of the frequency, attains a maximum. The obtained asymptotic formulae make possible to obtain an explicit expression of the peak frequency through the properties of the reservoir rock and the fluid. The range of the magnitude of this frequency predicted by the calculations is in agreement with the field observations [21,25]. The practical implications of the theory developed here are seismic modeling, inversion, and attribute analysis.

The technical approach employed in this study is based on a combination of the Hooke's law, momentum and mass balance equations, and the Darcy's law. Our derivation is different from that of Biot $[5,6]$. Although the obtained equations are essentially the same, we obtain new physical interpretations of some coefficients of the classical theory of poroelasticity. For instance, our derivation suggests that the Biot-Willis coefficient $\alpha$ [8] is related to the relative surface area of the grains, which is not exposed to 
the fluid. We demonstrate that the Gassmann's results [19] can be interpreted as the zero-frequency limit of the asymptotic solution presented in this study.

The total stress in a fluid-saturated porous medium includes the contact forces between the grains, the interactions between the solid and the fluid, and the fluid pressure. Fluid flow dissipates the energy through viscous friction. We assume that the deformations are small and the elastic properties of the skeleton are adequately described by the Hooke's law for a homogeneous isotropic elastic medium [38], whereas the fluid flow is governed by the dynamic Darcy's law [1]. We demonstrate that this modification of the Darcy's law is equivalent to a linearization of the law introduced in $[11,13,34]$ for a periodic oscillatory flow. It turns out that the relaxation time in the dynamic Darcy's law is associated with the coupling factor in Biot's model. One of our results is that the dynamic component of the Darcy's law does not affect the leading terms of the asymptotic expressions. In other words, the classical Darcy's law $[14,32,33]$ is sufficient for the low-frequency analysis.

The approach employed in the present work, to a certain degree, is an extension of the work [43]. Here, besides a slightly different choice of the small parameter, we abandon the assumption of grain stiffness employed in [43] and, in the study of reflection and transmission coefficients, we assume a permeable interface.

The paper is organized as follows. In Section 2, we briefly overview the Biot's theory of poroelasticity and derive the main equations. In Section 3, we obtain an asymptotic harmonic-wave solution valid in the low-frequency range. In Section 4 we obtain explicit expressions for the reflection and transmission coefficients for a planar compression wave crossing a permeable interface. The resonant frequency of a fast wave reflection from a permeable layer is studied in Section 5. Finally, Section 6 summarizes the findings and formulates the conclusions.

\section{THe Model}

In this section, we briefly overview the Biot's model of poroelasticity as an implication of the mass and momentum balance equations for a fluidsaturated medium. The porous medium is assumed to be homogeneous and isotropic, and the fluid is newtonian. The linear dimensions of an elementary volume of the medium are small relative to the linear dimensions of the entire medium, but large relative to the size of individual pores or grains constituting the solid skeleton.

We begin with the momentum balance equations for the bulk medium. The total stress is the resultant of three components: the elastic stress in the solid skeleton, the fluid pressure, and the viscous friction between the solid and the flowing fluid. In a linear approximation, these components are decoupled, so they can be considered separately. 
2.1. Linear elasticity of the skeleton. The Hooke's law describes the component of the total stress related to elastic deformations of the skeleton. Let us denote by $\mathbf{u}=\left(u_{x}, u_{y}, u_{z}\right)$ the displacement vector for the solid skeleton. The elements of the skeleton strain tensor, $\Xi=\left\{u_{i j}\right\}$, are defined as

$$
u_{11}=\frac{\partial u_{x}}{\partial x}, u_{12}=\frac{1}{2}\left(\frac{\partial u_{x}}{\partial y}+\frac{\partial u_{y}}{\partial x}\right), u_{13}=\frac{1}{2}\left(\frac{\partial u_{x}}{\partial z}+\frac{\partial u_{z}}{\partial x}\right), \text { etc }
$$

The trace of the strain tensor is equal to the divergence of the displacement vector, which, in turn, equals the volumetric strain. Thus, in the decomposition

$$
\Xi=\frac{1}{3} \nabla \cdot \mathbf{u} I+\left(\Xi-\frac{1}{3} \nabla \cdot \mathbf{u} I\right)
$$

the term in the brackets involves no volumetric strain. Here $I$ is an identity tensor. The Hooke's law for an isotropic and uniform medium determines the effective stress tensor:

$$
\sigma^{s}=K \nabla \cdot \mathbf{u} I+2 \mu\left(\Xi-\frac{1}{3} \nabla \cdot \mathbf{u} I\right)
$$

Here $K$ and $\mu$ are the bulk and shear moduli of the drained rock.

The macroscopic stress in a drained granular medium sums up from the grain-to-grain contact forces, Figure 1. Thus, Equation (3) involves a macroscopic-scale stress, that is a bulk-area average of the contact forces, and both moduli $K$ and $\mu$ are different from the respective moduli of the grain material.

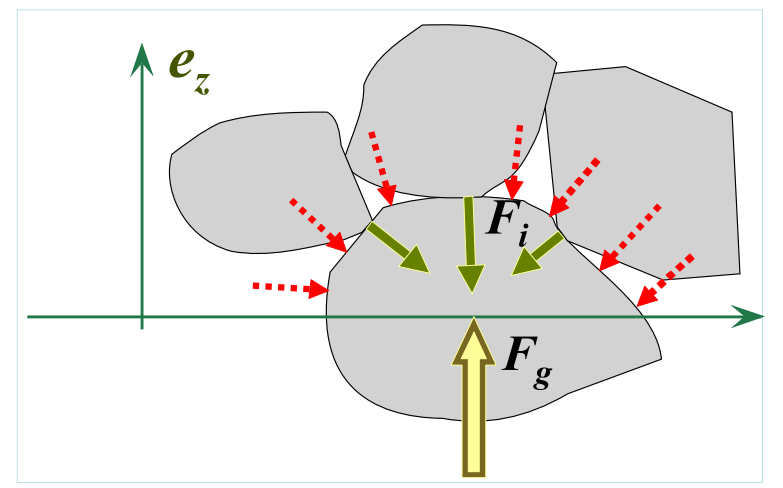

FiguRE 1. Force balance at an individual grain in a clean rock. The force $\mathbf{F}_{g}$ at a plane cross-section orthogonal to a unit normal $\mathbf{e}_{z}$ equals the sum of contact forces $\mathbf{F}_{i}$ shown as solid arrows, and fluid pressure acting at the part of the grain surface in located the positive with respect to $\mathbf{e}_{z}$ half-space, shown as dashed arrows. 


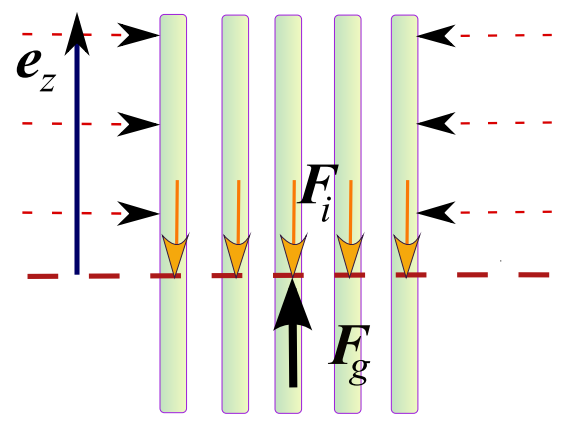

Figure 2. Colonnade-like skeleton: the action of fluid pressure shown by dashed arrows is orthogonal to the normal $\mathbf{e}_{z}$ to cross-section (bold dashed line). Therefore, the force $\mathbf{F}_{g}$ resulting from skeleton stress is the sum of co-directed individual forces $\mathbf{F}_{\mathbf{i}}$, and $\alpha_{\phi}=0$.

2.2. Fluid pressure. Consider the forces acting in the bulk medium at a plane cross-section, shown as the horizontal axis in Figure 1. The fluid pressure contribution in the total stress consists of two parts. In the fluid inside the pores, the total fluid pressure contribution amounts to $p \phi$, where $p$ denotes the pressure of the fluid and $\phi$ is the porosity of the medium. In the portions of the cross-section passing through the skeleton, the fluid pressure acts through the fluid-solid interactions at the surfaces of the grains. A portion of the surface at each grain is taken by the contacts with neighbor grains and is excluded from the fluid-solid interaction. Therefore, the total action of the fluid pressure on the skeleton is characterized by $(1-\phi) \alpha_{\phi} p$, where $\alpha_{\phi}$ is a dimensionless geometric factor accounting for the portion of the solid surface, which is excluded from a contact with the fluid. Thus, the entire pressure contribution into the total stress is

$$
\sigma^{p}=-\alpha p I
$$

where $\alpha=\phi+\alpha_{\phi}(1-\phi)$. The minus sign reflects the orientation convention. Clearly, $0 \leq \alpha_{\phi} \leq 1$, therefore $\phi \leq \alpha \leq 1$. The two extreme values of $\alpha$ are $\alpha=1$ and $\alpha=\phi$. The first one describes a medium where $\alpha_{\phi}=1$, which means that the grain-to-grain contact areas are negligibly small. The other extreme case implies $\alpha_{\phi}=0$. Such an equality takes place in a medium like the one shown in Figure 2, where the grains have the form of infinite spaghetti. The latter example is, apparently, a non-isotropic medium, and it is presented here for an illustration only.

The employed here pore-scale analysis of the stress descends from Khristianovich and Kovalenko [35].

2.3. Flow-imposed shear stress. Another stress component, which is a consequence of the fluid flow, is the shear stress due to the spacial variations of the Darcy velocity. To illustrate the micromechanics of this stress, consider two spherical grains immersed in fluid flow, Figure 3. The flow is along 


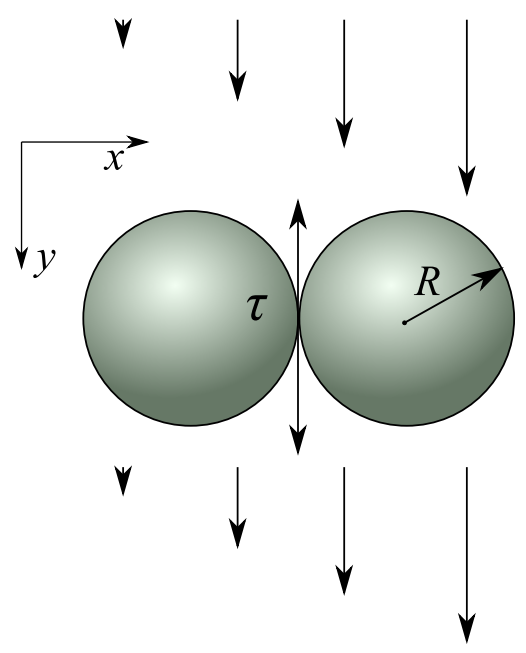

FiguRE 3. The viscose-friction imposed shear stress $\tau$ is the result of the spacial variations of the flow velocity.

the $y$ coordinate, and the tangential plane at the contact between the grains is orthogonal to the axis $x$. We assume that the flow velocity depends on $x$ only. In the creeping flow approximation, by virtue of the Stokes' formula, the viscous friction force acting on each grain is approximately equal to

$$
F_{g}=6 \pi R \eta V_{y}
$$

where $V_{y}$ is the mean flow velocity associated with the center of each sphere and $\eta$ is the viscosity of the fluid. Since $V_{y}$ depends on $x$, the forces acting on the left and the right grains are different. If the grains' motion relative to each other along the $y$ direction is constrained by friction or the contact cement, then the pair of forces at the contact develops a shear stress $\tau$. A rough estimate yields

$$
\tau \sim \frac{1}{4 \pi R^{2}} 6 \pi R \eta\left(V_{y}\left(x_{c}+R\right)-V_{y}\left(x_{c}-R\right)\right) \approx 3 \eta \frac{\partial V_{y}\left(x_{c}\right)}{\partial x}
$$

Here $x_{c}$ is the $x$ coordinate of the contact point. To evaluate the stress, the tangential contact force has been divided by the cross-section are of a sphere of radius $R$.

The calculation above is a great idealization. However, it suggests that in a shear planar wave, where the Darcy velocity is not constant along the wave front, the viscous friction will cause a shear stress, which is not associated with the deformation of the skeleton. To obtain the equations describing this stress, let us denote by $\mathbf{W}$ the Darcy velocity of the flowing fluid relative to the skeleton. The Darcy's law is, in particular, a manifestation of the friction forces between the flowing fluid and the solid $[32,33]$. The spacial variations of $\mathbf{W}$ imply spacial variations of these forces and impose a shear stress. At a uniform fluid flow, the shear stress vanishes. By analogy with the classical fluid mechanics [37], in a linear approximation in a homogeneous isotropic 
medium, the shear stress, $\sigma^{f}$, must be characterized by the gradient of the Darcy velocity.

$$
\sigma^{f}=-\varphi\left(\begin{array}{ccc}
0 & \frac{\partial W_{y}}{\partial x}+\frac{\partial W_{x}}{\partial y} & \frac{\partial W_{x}}{\partial z}+\frac{\partial W_{z}}{\partial x} \\
\frac{\partial W_{y}}{\partial x}+\frac{\partial W_{x}}{\partial y} & 0 & \frac{\partial W_{y}}{\partial z}+\frac{\partial W_{z}}{\partial y} \\
\frac{\partial W_{x}}{\partial z}+\frac{\partial W_{z}}{\partial x} & \frac{\partial W_{y}}{\partial z}+\frac{\partial W_{z}}{\partial y} & 0
\end{array}\right)
$$

The coefficient $\varphi$ must be a function of the fluid viscosity and the geometry of the pore space. Dimensional considerations suggest

$$
\varphi=\varphi_{0} \eta
$$

where $\varphi_{0}$ is a dimensionless shape factor.

2.4. Bulk momentum balance in a planar wave. In a linear approximation, the total stress, $\sigma^{t}$, is the sum of all three stresses defined in Equations (3), (4), and (7):

$$
\sigma^{t}=\sigma^{s}+\sigma^{p}+\sigma^{f}
$$

Let us denote by $\varrho_{g}$ the density of the solid constituent, which is the average density of the grains and cement material, and let $\varrho_{f}$ be the density of the fluid. Then, the bulk density is equal to

$$
\varrho_{b}=\phi \varrho_{f}+(1-\phi) \varrho_{g}
$$

Thus, the linearized momentum balance equation has the following from

$$
\varrho_{b} \frac{\partial^{2} \mathbf{u}}{\partial t^{2}}+\varrho_{f} \frac{\partial \mathbf{W}}{\partial t}=\nabla \cdot \sigma^{t}
$$

For a planar wave propagating in the direction $x$, all the derivatives with respect to $y$ and $z$ vanish, and Equations (11) reduce to

$$
\begin{aligned}
\varrho_{b} \frac{\partial^{2} u_{x}}{\partial t^{2}}+\varrho_{f} \frac{\partial W_{x}}{\partial t} & =M \frac{\partial^{2} u_{x}}{\partial x^{2}}-\alpha \frac{\partial p}{\partial x} \\
\varrho_{b} \frac{\partial^{2} u_{y}}{\partial t^{2}}+\varrho_{f} \frac{\partial W_{y}}{\partial t} & =\mu \frac{\partial^{2} u_{y}}{\partial x^{2}}-\varphi \frac{\partial^{2} W_{y}}{\partial x^{2}} \\
\varrho_{b} \frac{\partial^{2} u_{z}}{\partial t^{2}}+\varrho_{f} \frac{\partial W_{z}}{\partial t} & =\mu \frac{\partial^{2} u_{z}}{\partial x^{2}}-\varphi \frac{\partial^{2} W_{z}}{\partial x^{2}}
\end{aligned}
$$

where $M=K+\frac{4}{3} \mu$. 
2.5. Fluid flow momentum balance. The fluid flow relative to the skeleton is described by the dynamic Darcy's law

$$
\mathbf{W}+\tau \frac{\partial \mathbf{W}}{\partial t}=-\frac{\kappa}{\eta}\left(\nabla p+\varrho_{f} \frac{\partial^{2} \mathbf{u}}{\partial t^{2}}\right)
$$

Here $\kappa$ is the absolute permeability of the medium. The second-order time derivative of the skeleton displacement on the right-hand side of the last equation accounts for the fact that the reference system associated with the oscillating skeleton is not inertial. The relaxation time $\tau$ accounts both for the non-equilibrium effects of nonsteady fluid flow and the fluid inertia. A one-dimensional version of Darcy's law for a planar wave in the direction $x$ is straightforward.

2.6. Mass balance equations. The rock porosity and fluid density variations change the mass of fluid contained in an elementary volume. The rate of this change equals the total mass flux through the boundary of the volume. By retaining only the first-order terms, the mass balance for the fluid takes on the form:

$$
\phi \frac{\partial \varrho_{f}}{\partial t}+\varrho_{f} \frac{\partial \phi}{\partial t}=-\varrho_{f} \nabla \cdot\left(\mathbf{W}+\phi \frac{\partial \mathbf{u}}{\partial t}\right)
$$

Assuming linear fluid compressibility, one obtains

$$
\frac{d \varrho_{f}}{\varrho_{f}}=\beta_{f} d p
$$

We assume no heat flow, so $\beta_{f}$ is the coefficient of adiabatic compressibility of the fluid [37]. From Equations (14) and (15),

$$
\beta_{f} \phi \frac{\partial p}{\partial t}+\frac{\partial \phi}{\partial t}+\nabla \cdot \mathbf{W}+\phi \nabla \cdot \frac{\partial \mathbf{u}}{\partial t}=0
$$

The mass balance equation for the skeleton is:

$$
\frac{\partial\left((1-\phi) \varrho_{g}\right)}{\partial t}=-(1-\phi) \varrho_{g} \nabla \cdot \frac{\partial \mathbf{u}}{\partial t}
$$

or, equivalently,

$$
(1-\phi) \frac{1}{\varrho_{g}} \frac{\partial \varrho_{g}}{\partial t}-\frac{\partial \phi}{\partial t}=-(1-\phi) \nabla \cdot \frac{\partial \mathbf{u}}{\partial t}
$$

We assume that the compression of the grains is determined by the component of the skeleton stress coming from the volumetric strain (described by the term $K \nabla \cdot \mathbf{u}$ in Equations (2)-(3)) and the fluid pressure variations. In a linearized form, one obtains:

$$
\frac{1}{\varrho_{g}} d \varrho_{g}=-\frac{1}{K_{s g}} K \nabla \cdot \mathbf{u}+\frac{1}{K_{f g}} d p
$$


Here $K_{s g}$ and $K_{f g}$ are the elastic moduli quantifying the compression of the grains by the volumetric strain of the skeleton and the fluid pressure variations, respectively. Thus, for the time derivatives, one obtains

$$
\frac{1}{\varrho_{g}} \frac{\partial \varrho_{g}}{\partial t}=-\frac{K}{K_{s g}} \nabla \cdot \frac{\partial \mathbf{u}}{\partial t}+\frac{1}{K_{f g}} \frac{\partial p}{\partial t}
$$

The time derivative of the porosity can be eliminated from Equations (16) and (18):

$$
\beta_{f} \phi \frac{\partial p}{\partial t}+(1-\phi)\left[-\frac{K}{K_{s g}} \nabla \cdot \frac{\partial \mathbf{u}}{\partial t}+\frac{1}{K_{f g}} \frac{\partial p}{\partial t}\right]+\nabla \cdot \mathbf{W}+\nabla \cdot \frac{\partial \mathbf{u}}{\partial t}=0
$$

After gathering similar terms, the last equation, finally, takes on the form

$$
\frac{\gamma_{\beta}}{K} \frac{\partial p}{\partial t}+\gamma_{K} \nabla \cdot \frac{\partial \mathbf{u}}{\partial t}+\nabla \cdot \mathbf{W}=0
$$

where

$$
\gamma_{\beta}=K\left(\beta_{f} \phi+\frac{1-\phi}{K_{f g}}\right) \text { and } \gamma_{K}=1-\frac{(1-\phi) K}{K_{s g}}
$$

are dimensionless coefficients.

The skeleton is less stiff than the grain material: $K \leq K_{s g}$. Therefore,

$$
\phi \leq \gamma_{K} \leq 1
$$

At vanishing porosity, $\phi \rightarrow 0$, the skeleton strength approaches that of the grain material, $K / K_{s g} \rightarrow 1$. Therefore, if the transition to the zero porosity is smooth, which according to $[9,30]$ holds true for a variety of heterogeneous media, then

$$
\gamma_{K} \sim \phi \text { as } \phi \rightarrow 0
$$

The behavior of the coefficient $\gamma_{\beta}$ at $\phi \rightarrow 0$ depends, in particular, on the limit of the ratio $K / K_{f g}$. It is natural to assume that this ratio goes to zero as the porosity vanishes. However, besides the porosity, this ratio may also depend on the geometry of the pore space. In any case, the inequality $\gamma_{\beta} \geq$ $K \beta_{f} \phi$ must hold true. The compressibility of the fluid, $\beta_{f}$, is independent of the porosity, whereas the bulk modulus of the skeleton, $K$, converges to that of the grain material as $\phi \rightarrow 0$. Thus, Equations (23) and (25) imply

$$
\gamma_{\beta} \gg \gamma_{K}^{2} \text { as } \phi \rightarrow 0
$$

To compare the introduced coefficients to those of Biot and Willis [8], one can introduce a fluid displacement vector, w, as the integral of Darcy velocity:

$$
\mathbf{W}=\frac{d}{d t} \mathbf{w}
$$

Integration of Equation (22) with respect to $t$ yields

$$
p=-\frac{M \gamma_{K}}{\gamma_{\beta}} \nabla \cdot \mathbf{u}-\frac{M}{\gamma_{\beta}} \nabla \cdot \mathbf{w}
$$


The divergence in the last term, $\nabla \cdot \mathbf{w}$, is equal to the fluid content $[2,8,16]$. A comparison of the last equation to Equation [30] from [8] shows that

$$
\alpha_{B W}=\gamma_{K}, \text { and } M_{B W}=\frac{M}{\gamma_{\beta}}
$$

where the subscript $B W$ refers to the Biot-Willis coefficients in the notations of [8], and $\gamma_{K}$ and $\gamma_{\beta}$ are defined in Equation (23).

\section{Asymptotic haRmonic WAVE SOLUTion}

Let us seek a harmonic planar-wave solution to the obtained system of equations. That is, put

$$
\mathbf{u}=\mathbf{U}_{0} e^{i\left(\omega t-k_{x} x\right)}, \quad \mathbf{W}=\mathbf{W}_{0} e^{i\left(\omega t-k_{x} x\right)}, \text { and } p=p_{0} e^{i\left(\omega t-k_{x} x\right)}
$$

Here $k_{x}$ denotes the $x$-component of the complex-valued wave vector: $\mathbf{k}=$ $\left(k_{x}, 0,0\right)$. A substitution of Equation (30) into equations (12), (13), and (16) yields

$$
\begin{aligned}
-\omega^{2} \varrho_{b} U_{0 x}+i \omega \varrho_{f} W_{0 x} & =-M k_{x}^{2} U_{0 x}+i k_{x} \alpha p_{0} \\
-\omega^{2} \varrho_{b} U_{0 y}+i \varrho_{f} \omega W_{0 y} & =-\mu k_{x}^{2} U_{0 y}+\varphi k_{x}^{2} W_{0 y} \\
-\omega^{2} \varrho_{b} U_{0 z}+i \varrho_{f} \omega W_{0 z} & =-\mu k_{x}^{2} U_{0 z}+\varphi k_{x}^{2} W_{0 z} \\
W_{0 x}+i \omega \tau W_{0 x} & =\frac{\kappa}{\eta}\left(i k_{x} p_{0}+\omega^{2} \varrho_{f} U_{0 x}\right) \\
W_{0 y}+i \omega \tau W_{0 y} & =\frac{\kappa}{\eta} \omega^{2} \varrho_{f} U_{0 y} \\
W_{0 z}+i \omega \tau W_{0 z} & =\frac{\kappa}{\eta} \omega^{2} \varrho_{f} U_{0 z} \\
i \omega \frac{\gamma_{\beta}}{M} p_{0}+\gamma_{K} k_{x} \omega U_{0 x} & =i k_{x} W_{0 x}
\end{aligned}
$$

Like in the classical case, the compression and shear waves decouple and each component of the solution can be calculated separately.

3.1. Compression wave. Only those equations (31), which involve the $x$ components of the skeleton displacement and the Darcy velocity, constitute the system of compression wave equations:

$$
\begin{aligned}
-\omega^{2} \varrho_{b} U_{0 x}+i \varrho_{f} \omega W_{0 x} & =-M k_{x}^{2} U_{0 x}+i k_{x} \alpha p_{0} \\
W_{0 x}+i \omega \tau W_{0 x} & =\frac{\kappa}{\eta}\left(i k_{x} p_{0}+\omega^{2} \varrho_{f} U_{0 x}\right) \\
i \omega \frac{\gamma_{\beta}}{M} p_{0}+\gamma_{K} k_{x} \omega U_{0 x} & =i k_{x} W_{0 x}
\end{aligned}
$$

The equation

$$
\varepsilon=i \lambda \omega
$$

defines a dimensionless small parameter, where the quantity

$$
\lambda=\varrho_{f} \frac{\kappa}{\eta}
$$


can be called a kinematic reservoir fluid mobility. Let us define the dimensionless parameters by putting

$$
\zeta=\frac{v^{2}}{v_{f}^{2}}, \quad \xi=-i \frac{p_{0}}{k_{x} M U_{0 x}}, \text { and } \chi=i \frac{W_{0 x}}{\omega U_{0 x}}
$$

Here

$$
v^{2}=\frac{\omega^{2}}{k_{x}^{2}}, \quad \text { and } \quad v_{f}^{2}=\frac{M}{\varrho_{f}}
$$

Then, the system of equations (32) takes on the form:

$$
\begin{aligned}
\gamma_{\varrho} \zeta-\zeta \chi-\alpha \xi & =1 \\
\varepsilon(\zeta-\vartheta \zeta \chi-\xi)-\zeta \chi & =0 \\
\gamma_{\beta} \xi+\chi & =\gamma_{K}
\end{aligned}
$$

where

$$
\gamma_{\varrho}=\frac{\varrho_{b}}{\varrho_{f}} \text { and } \vartheta=\frac{\tau}{\lambda}
$$

Even though both parameters, $v$ and $v_{f}$, have dimensions of velocity, they do not have immediate physical interpretation.

We seek a solution to the system (37)-(39) in the form of power series in $\varepsilon:$

$$
\begin{aligned}
& \zeta=\zeta_{0}+\zeta_{1} \varepsilon+\zeta_{2} \varepsilon^{2}+\ldots \\
& \xi=\xi_{0}+\xi_{1} \varepsilon+\xi_{2} \varepsilon^{2}+\ldots
\end{aligned} \quad \chi=\chi_{0}+\chi_{1} \varepsilon+\chi_{2} \varepsilon^{2}+\ldots
$$

where "..." denotes the higher order terms. The coefficients in these power series can be determined by a substitution of Equations (41) in (37)-(39), and equating the terms with similar powers of $\varepsilon$. In particular, one infers:

$$
\frac{\omega^{2}}{k_{x}^{2} v_{f}^{2}}=\zeta_{0}+\zeta_{1} \varepsilon+\zeta_{2} \varepsilon^{2}+\ldots
$$

A harmonic-wave solution in the form of Equation (32) is physically sensible only if the real part of $k_{x}$ is positive, and the imaginary part is negative. Therefore, the first two coefficients in the power-seris expansion for $\zeta, \zeta_{0}$ and $\zeta_{1}$, must be positive. We will see below that this observation will lead to a coupling relationship between $\gamma_{K}$ and $\alpha$.

At $\varepsilon=0$, Equation (38) implies $\zeta_{0} \chi_{0}=0$. One obtains two zero-order solutions:

$$
\begin{aligned}
\zeta_{0}^{S} & =0 & \zeta_{0}^{F} & =\frac{\alpha \gamma_{K}+\gamma_{\beta}}{\gamma_{\beta} \gamma_{\varrho}} \\
\xi_{0}^{S} & =-\frac{1}{\alpha} & \xi_{0}^{F} & =\frac{\gamma_{K}}{\gamma_{\beta}} \\
\chi_{0}^{S} & =\gamma_{K}+\frac{\gamma_{\beta}}{\alpha} & \chi_{0}^{F} & =0
\end{aligned}
$$


The superscripts ${ }^{S}$ and ${ }^{F}$ stand for the slow and fast waves. To determine the first-order terms, one has to solve the following system of equations:

$$
\begin{aligned}
\gamma_{\varrho} \zeta_{1}-\zeta_{0} \chi_{1}-\chi_{0} \zeta_{1}-\alpha \xi_{1} & =0 \\
\zeta_{0} \chi_{1}+\chi_{0} \zeta_{1} & =\zeta_{0}-\vartheta \chi_{0} \zeta_{0}-\xi_{0} \\
\gamma_{\beta} \xi_{1}+\chi_{1} & =0
\end{aligned}
$$

Note that $\vartheta$ is the only dimensionless parameter depending on the relaxation time $\tau$, see Equation (40). Since $\chi_{0} \zeta_{0}=0$ both for the slow-wave and the fast-wave solutions, the only term involving $\vartheta$ in the second Equation (44) vanishes. Consequently, the classical steady-state Darcy's law formulation is sufficient for the first-order asymptotic analysis.

The solution to the system of equations (44) is:

$$
\begin{aligned}
\zeta_{1}^{S} & =\frac{1}{\alpha \gamma_{K}+\gamma_{\beta}} & \zeta_{1}^{F} & =\frac{\zeta_{0}^{F}-\frac{\alpha}{\gamma_{\beta}}}{\gamma_{\varrho} \zeta_{0}^{F}}\left(\zeta_{0}^{F}-\xi_{0}^{F}\right) \\
\xi_{1}^{S} & =\frac{1}{\alpha} \frac{\gamma_{\varrho}}{\alpha \gamma_{K}+\gamma_{\beta}}-\frac{1}{\alpha} & \xi_{1}^{F} & =\frac{\gamma_{K} \gamma_{\varrho}}{\left(\alpha \gamma_{K}+\gamma_{\beta}\right) \gamma_{\beta}}-\frac{1}{\gamma_{\beta}} \\
\chi_{1}^{S} & =-\frac{1}{\alpha} \frac{\gamma_{\varrho} \gamma_{\beta}}{\alpha \gamma_{K}+\gamma_{\beta}}+\frac{\gamma_{\beta}}{\alpha} & \chi_{1}^{F} & =1-\frac{\gamma_{K} \gamma_{\varrho}}{\alpha \gamma_{K}+\gamma_{\beta}}
\end{aligned}
$$

From equations (43), $\zeta_{0}^{F}>0$. By the remark after Equation (42), $\zeta_{1}^{F}$ must be positive. This requirement is fulfilled for an arbitrary $\zeta_{0}^{F}$ only if $\frac{\alpha}{\gamma_{\beta}}=\xi_{0}^{F}$. Substituting the expression for $\xi_{0}^{F}$ from Equation (43), one obtains:

$$
\alpha=\gamma_{K}=1-\frac{(1-\phi) K}{K_{s g}}
$$

Now, Equations (43) and (45) can be rewritten in the final form:

$$
\begin{aligned}
\zeta_{0}^{S} & =0 & \zeta_{0}^{F} & =\frac{\gamma_{K}^{2}+\gamma_{\beta}}{\gamma_{\beta} \gamma_{\varrho}} \\
\xi_{0}^{S} & =-\frac{1}{\gamma_{K}} & \xi_{0}^{F} & =\frac{\gamma_{K}}{\gamma_{\beta}} \\
\chi_{0}^{S} & =\frac{\gamma_{K}^{2}+\gamma_{\beta}}{\gamma_{K}} & \chi_{0}^{F} & =0
\end{aligned}
$$

and

$$
\begin{aligned}
\zeta_{1}^{S} & =\frac{1}{\gamma_{K}^{2}+\gamma_{\beta}} & \zeta_{1}^{F} & =\frac{1}{\gamma_{\beta}\left(\gamma_{K}^{2}+\gamma_{\beta}\right)}\left(\frac{\gamma_{K}^{2}+\gamma_{\beta}}{\gamma_{\varrho}}-\gamma_{K}\right)^{2} \\
\xi_{1}^{S} & =\frac{1}{\gamma_{K}} \frac{\gamma_{\varrho}}{\gamma_{K}^{2}+\gamma_{\beta}}-\frac{1}{\gamma_{K}} & \xi_{1}^{F} & =\frac{\gamma_{K} \gamma_{\varrho}}{\left(\gamma_{K}^{2}+\gamma_{\beta}\right) \gamma_{\beta}}-\frac{1}{\gamma_{\beta}} \\
\chi_{1}^{S} & =-\frac{1}{\gamma_{K}} \frac{\gamma_{\varrho} \gamma_{\beta}}{\gamma_{K}{ }^{2}+\gamma_{\beta}}+\frac{\gamma_{\beta}}{\gamma_{K}} & \chi_{1}^{F} & =1-\frac{\gamma_{K} \gamma_{\varrho}}{\gamma_{K}^{2}+\gamma_{\beta}}
\end{aligned}
$$


From the leftmost equation (35),

$$
k_{x}^{2}=\frac{\varrho_{f}}{M \zeta} \omega^{2}
$$

Hence, for the fast wave,

$$
k_{x}^{F}=\frac{\omega}{v_{b}} \sqrt{\frac{\gamma_{\beta}}{\gamma_{\beta}+\gamma_{K}^{2}}}\left(1-\frac{\zeta_{1}^{F}}{2 \zeta_{0}^{F}} \varepsilon+O\left(|\varepsilon|^{2}\right)\right)
$$

where $v_{b}^{2}=\frac{M}{\varrho_{b}}$. For the slow wave,

$$
k_{x}^{S}=\frac{\omega}{v_{f}} \sqrt{\frac{\gamma_{K}^{2}+\gamma_{\beta}}{2|\varepsilon|}}(1-i)(1+O(|\varepsilon|))
$$

In the last equation, the branch of the square root has been selected to guarantee that $\operatorname{Im}\left(k_{x}^{S}\right)<0$, that is,

$$
\sqrt{\varepsilon}=\frac{1+i}{\sqrt{2}} \sqrt{|\varepsilon|}=\sqrt{|\varepsilon|} e^{i \frac{\pi}{4}}
$$

Equations (50) and (51) can be rearranged into the from:

$$
\begin{aligned}
& k_{x}^{F}=\omega\left(k_{0}^{F}+k_{1}^{F} \varepsilon+O\left(|\varepsilon|^{2}\right)\right) \\
& k_{x}^{S}=\omega\left(k_{0}^{S} \frac{1}{\sqrt{\varepsilon}}+k_{1}^{S} \sqrt{\varepsilon}+O\left(|\varepsilon|^{3 / 2}\right)\right)
\end{aligned}
$$

where

$$
k_{0}^{F}=\frac{1}{v_{b}} \sqrt{\frac{\gamma_{\beta}}{\gamma_{\beta}+\gamma_{K}^{2}}} \text { and } k_{0}^{S}=\frac{1}{v_{f}} \sqrt{\gamma_{\beta}+\gamma_{K}^{2}}
$$

The estimate in Equation (26) implies

$$
k_{0}^{F} \approx \frac{1}{v_{b}} \text { as } \phi \rightarrow 0
$$

The velocities and attenuation factors for the fast and slow waves are

$$
\begin{aligned}
V^{F} & =v_{b} \sqrt{1+\frac{\gamma_{K}^{2}}{\gamma_{\beta}}}\left(1+O\left(|\varepsilon|^{2}\right)\right) \\
V^{S} & =v_{f} \sqrt{\frac{2|\varepsilon|}{\gamma_{\beta}+\gamma_{K}^{2}}}(1+O(|\varepsilon|)) \\
a^{F} & =\frac{\omega}{v_{b}} \sqrt{\frac{\gamma_{\beta}}{\gamma_{\beta}+\gamma_{K}^{2}}} \frac{\zeta_{1}^{F}}{2 \zeta_{0}^{F}}|\varepsilon|\left(1+O\left(|\varepsilon|^{2}\right)\right) \\
a^{S} & =\frac{\omega}{v_{f}} \sqrt{\frac{\gamma_{\beta}+\gamma_{K}^{2}}{2|\varepsilon|}}(1+O(|\varepsilon|))
\end{aligned}
$$


By the definition of $\varepsilon$ in Equation (33), the angular frequency $\omega$ is present both in the numerator and the denominator in the last equation. It is useful to rewrite it in the alternative from

$$
a^{S}=\frac{\eta}{\kappa} \sqrt{\frac{\gamma_{\beta}+\gamma_{K}^{2}}{2 M \varrho_{f}}} \sqrt{|\varepsilon|}
$$

Clearly, $V^{F} \gg V^{S}=O(\sqrt{|\varepsilon|})$ and $a^{F} \ll a^{S}$ as $\varepsilon \rightarrow 0$. Equation (25) also implies that, for $\phi \rightarrow 0$, the fast compressive wave velocity approaches the velocity of sound in a medium whose density equals the density of the grains and elastic moduli are those of the drained skeleton.

The Darcy velocity and the fluid pressure have the following asymptotic forms:

$$
\begin{aligned}
W_{0 x}^{F} & =-i \omega \varepsilon\left(\chi_{1}^{F}+O(|\varepsilon|)\right) U_{0 x}^{F} \\
W_{0 x}^{S} & =-i \omega \frac{\gamma_{\beta}+\gamma_{K}^{2}}{\gamma_{K}}\left(1+\frac{\chi_{1}^{S}}{\chi_{0}^{S}} \varepsilon+O\left(|\varepsilon|^{2}\right)\right) U_{0 x}^{S} \\
p_{0}^{F} & =\omega\left[k_{0}^{F}+\left(k_{1}^{F} \xi_{0}^{F}+k_{0}^{F} \xi_{1}^{F}\right) \varepsilon+O\left(|\varepsilon|^{2}\right)\right] M U_{0 x}^{F} \\
p_{0}^{S} & =\frac{\omega}{\sqrt{|\varepsilon|}}\left[k_{0}^{S}+\left(k_{1}^{S} \xi_{0}^{S}+k_{0}^{S} \xi_{1}^{S}\right) \varepsilon+O\left(|\varepsilon|^{2}\right)\right] M U_{0 x}^{S}
\end{aligned}
$$

3.2. Shear wave. The shear waves in the directions $y$ and $z$ are analogous to each other, so we consider in detail only the shear wave in the direction $y$. Since there is no volume variation in a shear deformation, the mass balance equation is an identity. Thus, only the following system of two equations needs be solved:

$$
\begin{aligned}
-\omega^{2} \varrho_{b} U_{0 y}+i \varrho_{f} \omega W_{0 y} & =\mu k_{x}^{2} U_{0 y}-\chi k_{x}{ }^{2} W_{0 y} \\
W_{0 y}+i \omega \tau W_{0 y} & =\frac{\kappa}{\eta} \omega^{2} \varrho_{f} U_{0 y}
\end{aligned}
$$

A convenient set of dimensionless variables is provided be the first and last equations (35), where the $x$-components are replaced with the respective $y$-components. The system of equations (66) transforms into the following:

$$
\begin{array}{r}
\gamma_{\varrho} \zeta-\zeta \chi+\varepsilon \frac{\chi \eta}{\mu \varrho_{f} \kappa} \chi=1 \\
\chi+\varepsilon \vartheta \chi=\varepsilon
\end{array}
$$

which is equivalent to Equations $(37)-(38)$ at $\xi=0$ with $M$ replaced with $\mu$. The unique solution to the system (67) is:

$$
\zeta=\frac{1-\frac{\varepsilon^{2}}{1+\varepsilon \vartheta} \frac{\chi \eta}{\mu \varrho_{f} \kappa}}{\gamma_{\varrho}-\frac{\varepsilon}{1+\varepsilon \vartheta}} \text { and } \chi=\frac{\varepsilon}{1+\varepsilon \vartheta}
$$


In the form of a power series in $\varepsilon$, this solution takes on the form:

$$
\begin{aligned}
\zeta & =\frac{1}{\gamma_{\varrho}}+\frac{1}{\gamma_{\varrho}^{2}} \varepsilon+O\left(|\varepsilon|^{2}\right) \\
\chi & =\varepsilon\left(1-\vartheta \varepsilon+O\left(|\varepsilon|^{2}\right)\right)
\end{aligned}
$$

The absence of the slow wave component in the shear wave solution is an implication of the fact that the fluid pressure diffusion is one-dimensional, in the direction $x$ only.

Returning back to the physical quantities, one obtains

$$
k_{x}^{H}=\sqrt{\frac{\varrho_{b}}{\mu}} \omega\left(1-\frac{1}{2 \gamma_{\varrho}} \varepsilon+O\left(|\varepsilon|^{2}\right)\right)
$$

where the superscript ${ }^{H}$ denotes a shear wave. Thus,

$$
k^{H}=\omega\left(k_{0}^{H}+k_{1}^{H} \varepsilon+O\left(|\varepsilon|^{2}\right)\right),
$$

where $k_{0}^{H}=\sqrt{\varrho_{b} / \mu}$. For the attenuation factor, $a^{H}$, and the velocity, $V^{H}$, one infers

$$
\begin{aligned}
a^{H} & =\sqrt{\frac{\varrho_{b}}{\mu}} \frac{|\varepsilon|}{2 \gamma_{\varrho}} \omega\left(1+O\left(|\varepsilon|^{2}\right)\right) \\
V^{H} & =\sqrt{\frac{\mu}{\varrho_{b}}}\left(1+O\left(|\varepsilon|^{2}\right)\right)
\end{aligned}
$$

3.3. Further remarks. The parameters and coefficients used in this study look not necessarily the same as those traditionally used in the theory of poroelasticity. In this section, we demonstrate that there is no contradiction. Moreover, the approach developed here provides new physical interpretations of the traditionally used parameters.

3.3.1. The dynamic Darcy's law and the flow-imposed shear stress. For an oscillatory fluid flow in a porous medium, Johnson et al. [34] have obtained a modification of the Darcy's law, in which the coefficient of permeability depends on the frequency of the oscillations:

$$
W=-\frac{\widetilde{k}(\omega)}{\eta} \nabla P
$$

At the zero frequency limit, the frequency-dependent coefficient of permeability must reduce to the classical Darcy permeability: $\widetilde{k}(0)=k$. Therefore, one can write:

$$
A(i \omega) \widetilde{k}(\omega)=k \text { with } A(0)=1
$$

Using Taylor expansion, $A(i \omega)=1+A^{\prime}(0) i \omega+\ldots$, and truncating the higher-order terms, one obtains from Equation (74):

$$
W+A^{\prime}(0) i \omega W=-\frac{k}{\eta} \nabla P
$$

The last equation is equivalent to Equation (13) for $\tau=A^{\prime}(0)$. 
Equations (43), (45), and (69) imply that both, the dynamic term in the dynamic Darcy's law and the flow-induced shear stress, affect only the power series terms of the order of $O\left(|\varepsilon|^{2}\right)$ or higher. Thus, the classical steady-state formulation of the Darcy's law is sufficient for the first-order asymptotic approximation of the compression wave solution. However, from Equation (68), the coefficients of the power series for the shear wave solution involve the viscosity of the fluid as a factor. Hence, for a very viscous fluid and finite values of $\varepsilon$, the higher-order terms of the power series may become comparable to the leading terms.

3.3.2. Elastic moduli. The compressibility of the fluid and the moduli of drained skeleton can be determined in laboratory tests. The other two moduli introduces in Equation (19) also can be determined experimentally. For the first test, one can change the pore pressure by injecting or withdrawing of fluid while maintaining the total stress constant. From the fluid compressibility and the variation of the fluid volume, one can evaluate the variation of the pore volume and, consequently, the variation of the total volume of the solid grains. Hence, from the known mass of the solid skeleton one obtains the variation of the average density of the grains. In an undrained uniaxial test, one also can measure the variation of the fluid pressure and the total volume of the saturated sample. Therefore, the variation of the average density of the grains can be calculated as well. Thus, knowing $K$ from independent measurements, one obtains a system of two equations, which can be solved for the moduli $K_{s g}$ and $K_{f g}$.

3.3.3. Gassmann's model. The two elastic moduli, $K_{s g}$ and $K_{f g}$, defined in Equation (19) relate the grain volumetric strain to the skeleton stress and fluid pressure, respectively. For each grain, these stresses act only on a part of the surface area. Therefore, one can expect that the same stress applied on a part of the area causes a smaller volume variation than if it were applied over the entire surface. Thus,

$$
K_{g} \leq K_{s g} \quad \text { and } \quad K_{g} \leq K_{f g}
$$

If the relationships

$$
K_{s g}=\frac{K_{g}}{1-\phi} \quad \text { and } \quad K_{f g}=\frac{K_{g}}{1-\frac{K}{K_{g}}}
$$

hold true, then the first equation (57) evaluated at a zero frequency yields the Gassmann's modulus [19, 39].

Proposition 42 in [19] indicates that the coefficient $\alpha$ is assumed to be equal to one. The asymptotic analysis presented above shows that $\alpha=1$ may lead to a non-physical negative attenuation factor for the fast-wave solution, unless $K_{s g} \gg K$. 
3.3.4. Biot's equations. Equations (46) and (29) show that the coefficient $\alpha$ introduced in Section (4) and the Biot-Willis coefficients alpha are the same. Further on, Equation (28) makes possible to eliminate the fluid pressure $p$ from the first Equation (12) and Equation (13). Thus, using the notation (27), one obtains:

$$
\begin{gathered}
\varrho_{b} \frac{\partial^{2} u_{x}}{\partial t^{2}}+\varrho_{f} \frac{\partial^{2} w_{x}}{\partial t^{2}}=M\left(1+\frac{\gamma_{K}^{2}}{\gamma_{\beta}}\right) \frac{\partial^{2} u_{x}}{\partial x^{2}}+\frac{M \gamma_{K}}{\gamma_{\beta}} \frac{\partial^{2} w}{\partial x^{2}} \\
\varrho_{f} \frac{\partial^{2} u_{x}}{\partial t^{2}}+\tau \frac{\eta}{\kappa} \frac{\partial^{2} w_{x}}{\partial t^{2}}=\frac{M \gamma_{K}}{\gamma_{\beta}} \frac{\partial^{2} u_{x}}{\partial x^{2}}+\frac{M}{\gamma_{\beta}} \frac{\partial^{2} w}{\partial x^{2}}-\frac{\eta}{\kappa} \frac{\partial w_{x}}{\partial t}
\end{gathered}
$$

The last system of equations is equivalent to the Biot's equations [7] with the following mapping rule:

$$
\begin{aligned}
A & =M\left(1+\frac{\gamma_{K}^{2}}{\gamma_{\beta}}\right) \\
M_{11} & =M \frac{\gamma_{K}}{\gamma_{\beta}} \\
M & =M \frac{1}{\gamma_{\beta}} \\
m & =\tau \frac{\eta}{\kappa}
\end{aligned}
$$

Here the notations and the parameters on the left-hand sides of equations (81) are from [7].

3.3.5. Vanishing attenuation. For some combinations of parameters, the coefficient $\zeta_{1}^{F}$ in Equation (45) can vanish. In such a case, the first-order approximation of the the fast wave attenuation is equal to zero. For example, $\zeta_{1}^{F}=0$ if $\gamma_{K}=\sqrt{\gamma_{\beta}}$ and $\gamma_{\varrho}=2 \gamma_{K}$ simultaneously.

\section{Normal REFLECTION OF A COMPRESSiON WAVE}

4.1. Fast incident wave. Let two media, whose properties will be labelled by the superscripts ${ }^{1}$ and ${ }^{2}$, have a permeable plane interface at $x=0$. Then an incident fast wave arriving from the half-space $x<0$ generates four waves: the fast and slow reflected waves and the fast and slow transmitted waves. Thus, in the medium 1, the displacement can be characterized as

$$
u_{1}(t, x)=U_{0} e^{i\left(\omega t-k^{1 F} x\right)}+R^{F F} U_{0} e^{i\left(\omega t+k^{1 F} x\right)}+R^{F S} U_{0} e^{i\left(\omega t+k^{1 S} x\right)}
$$

whereas in medium 2 , the skeleton displacement is

$$
u_{2}(t, x)=T^{F F} U_{0} e^{i\left(\omega t-k^{2 F} x\right)}+T^{F S} U_{0} e^{i\left(\omega t-k^{2 S} x\right)}
$$

Here $R^{F F}, R^{F S}, T^{F F}$, and $T^{F S}$ are the respective reflection and transmission coefficients. The first letter in the superscript denotes the fast incident wave, whereas the second one denotes the fast or slow reflected or transmitted wave. 
The mass and momentum balance imply that the skeleton displacement, the Darcy velocity of the fluid, the total stress, and the fluid pressure must be continuous at the interface. Using the notations (35), one obtains the following system of boundary conditions

$$
\begin{gathered}
1+R^{F F}+R^{F S}=T^{F F}+T^{F S} \\
\chi^{1 F}\left(1+R^{F F}\right)+\chi^{1 S} R^{F S}=\chi^{2 F} T^{F F}+\chi^{2 S} T^{F S} \\
M^{1} k^{1 F}\left(1+\gamma_{K}^{1} \xi^{1 F}\right)\left(1-R^{F F}\right)-M^{1} k^{1 S}\left(1+\gamma_{K}^{1} \xi^{1 S}\right) R^{F S} \\
=M^{2} k^{2 F}\left(1+\gamma_{K}^{2} \xi^{2 F}\right) T^{F F}+M^{2} k^{2 S}\left(1+\gamma_{K}^{2} \xi^{2 S}\right) T^{F S} \\
M^{1} k^{1 F} \xi^{1 F}\left(1-R^{F F}\right)-M^{1} k^{1 S} \xi^{1 S} R^{F S} \\
=M^{2} k^{2 F} \xi^{2 F} T^{F F}+M^{2} k^{2 S} \xi^{2 S} T^{F S}
\end{gathered}
$$

To obtain asymptotic expressions for the reflection and transmission coefficients, we rewrite Equations (84)-(87) in an asymptotic form, retaining only the zero-order and the next after zero-order terms. For the first two equations, it means the constant and linear in $\varepsilon$ terms, whereas for the last two equations, those terms are the constant and the ones proportional to $\sqrt{|\varepsilon|}$. One obtains:

$$
\begin{gathered}
1+R^{F F}+R^{F S}=T^{F F}+T^{F S} \\
\varepsilon \chi_{1}^{1 F}\left(1+R^{F F}\right)+\left(\chi_{0}^{1 S}+\varepsilon \chi_{1}^{1 S}\right) R^{F S} \\
=\gamma_{\kappa} \varepsilon \chi_{1}^{2 F} T^{F F}+\left(\chi_{0}^{2 S}+\chi_{1}^{2 S} \gamma_{\kappa} \varepsilon\right) T^{F S} \\
\sqrt{\varepsilon} M^{1} k_{0}^{1 F}\left(1+\gamma_{K}^{1} \xi_{0}^{1 F}\right)\left(1-R^{F F}\right)-M^{1} k_{0}^{1 S}\left(1+\gamma_{K}^{1} \xi_{0}^{1 S}\right) R^{F S} \\
=\sqrt{\varepsilon} M^{2} k_{0}^{2 F}\left(1+\gamma_{K}^{2} \xi_{0}^{2 F}\right) T^{F F}+\frac{1}{\sqrt{\gamma_{\kappa}}} M^{2} k_{0}^{2 S}\left(1+\gamma_{K}^{2} \xi_{0}^{2 S}\right) T^{F S} \\
\sqrt{\varepsilon} M^{1} k_{0}^{1 F} \xi_{0}^{1 F}\left(1-R^{F F}\right)-M^{1} k_{0}^{1 S} \xi_{0}^{1 S} R^{F S} \\
=\sqrt{\varepsilon} M^{2} k_{0}^{2 F} \xi_{0}^{2 F} T^{F F}+\frac{1}{\sqrt{\gamma_{\kappa}}} M^{2} k_{0}^{2 S} \xi_{0}^{2 S} T^{F S}
\end{gathered}
$$

Here

$$
\gamma_{\kappa}=\frac{\varepsilon_{2}}{\varepsilon_{1}}=\frac{\kappa_{2}}{\kappa_{1}}=1+\frac{\kappa_{2}-\kappa_{1}}{\kappa_{1}}
$$

By virtue of Equations (47),

$$
1+\gamma_{K}^{i} \xi_{0}^{i F}=\frac{\gamma_{\beta}^{i}+\left(\gamma_{K}^{i}\right)^{2}}{\gamma_{\beta}^{i}} \quad \text { and } \quad 1+\gamma_{K}^{i} \xi_{0}^{1 S}=0, \quad i=1,2
$$

Thus, the slow-wave reflection and transmission terms vanish in Equation (90). Taking into account Equation (55), Equation (90) reduces to

$$
Z_{1}\left(1-R^{F F}\right)=Z_{2} T^{F F}
$$


where $Z^{i}, i=1,2$, are the modified acoustic impedances:

$$
Z_{i}=\frac{M^{i}}{v_{b}^{i}} \sqrt{\frac{\gamma_{\beta}^{i}+\left(\gamma_{K}^{i}\right)^{2}}{\gamma_{\beta}^{i}}}
$$

We seek asymptotic expressions for the transmission and reflection coefficients in the form

$$
R=R_{0}+R_{1} \sqrt{\varepsilon}+R_{2} \varepsilon+\ldots \text { and } T=T_{0}+T_{1} \sqrt{\varepsilon}+T_{2} \varepsilon+\ldots
$$

We limit our calculations to the first two terms only. Putting $\varepsilon=0$ yields:

$$
\begin{aligned}
& R_{0}^{F S}=T_{0}^{F S}=0 \\
& R_{0}^{F F}=\frac{Z_{1}-Z_{2}}{Z_{1}+Z_{2}} \text { and } T_{0}^{F F}=\frac{2 Z_{1}}{Z_{1}+Z_{2}}
\end{aligned}
$$

Equations (89) and (91) imply

$$
\begin{gathered}
\frac{\left(\gamma_{K}^{1}\right)^{2}+\gamma_{\beta}^{1}}{\gamma_{K}^{1}} R_{1}^{F S}-\frac{\left(\gamma_{K}^{2}\right)^{2}+\gamma_{\beta}^{2}}{\gamma_{K}^{2}} T_{1}^{F S}=0 \\
\frac{M^{1}}{v_{F}^{1}} \frac{\sqrt{\left(\gamma_{K}^{1}\right)^{2}+\gamma_{\beta}^{1}}}{\gamma_{K}^{1}} R_{1}^{F S}+\frac{1}{\sqrt{\gamma_{\kappa}}} \frac{M^{2}}{v_{F}^{2}} \frac{\sqrt{\left(\gamma_{K}^{2}\right)^{2}+\gamma_{\beta}^{2}}}{\gamma_{K}^{2}} T_{1}^{F S} \\
=M^{1} k_{0}^{1 F} \xi_{0}^{1 F}\left(1-R_{0}^{F F}\right)-M^{2} k_{0}^{2 F} \xi_{0}^{2 F} T_{0}^{F F}
\end{gathered}
$$

The right-hand side of the last equation can be written in the form

$$
A=\left[\frac{\gamma_{K}^{1}}{\left(\gamma_{K}^{1}\right)^{2}+\gamma_{\beta}^{1}}-\frac{\gamma_{K}^{2}}{\left(\gamma_{K}^{2}\right)^{2}+\gamma_{\beta}^{2}}\right] \frac{2 Z_{1} Z_{2}}{Z_{1}+Z_{2}}
$$

The determinant of the linear system of equations (99)-(100) equals

$$
\begin{aligned}
& D=\frac{1}{\sqrt{\gamma_{\kappa}}} \frac{M^{2}}{v_{F}^{2}} \frac{\left(\gamma_{K}^{1}\right)^{2}+\gamma_{\beta}^{1}}{\gamma_{K}^{1}} \frac{\sqrt{\left(\gamma_{K}^{2}\right)^{2}+\gamma_{\beta}^{2}}}{\gamma_{K}^{2}} \\
&+\frac{M^{1}}{v_{F}^{1}} \frac{\left(\gamma_{K}^{2}\right)^{2}+\gamma_{\beta}^{2}}{\gamma_{K}^{2}} \frac{\sqrt{\left(\gamma_{K}^{1}\right)^{2}+\gamma_{\beta}^{1}}}{\gamma_{K}^{1}}
\end{aligned}
$$

In particular, the determinant is obviously positive: $D>0$. Hence, one obtains

$$
R_{1}^{F S}=\frac{A}{D} \frac{\left(\gamma_{K}^{2}\right)^{2}+\gamma_{\beta}^{2}}{\gamma_{K}^{2}} \text { and } T_{1}^{F S}=\frac{A}{D} \frac{\left(\gamma_{K}^{1}\right)^{2}+\gamma_{\beta}^{1}}{\gamma_{K}^{1}}
$$

For the next terms of the fast wave reflection and transmission coefficients, one obtains

$$
\begin{aligned}
R_{1}^{F F}-T_{1}^{F F} & =T_{1}^{F S}-R_{1}^{F S} \\
Z_{1} R_{1}^{F F}+Z^{2} T_{1}^{F F} & =0
\end{aligned}
$$


and

$$
R_{1}^{F F}=\frac{Z_{2}\left(T_{1}^{F S}-R_{1}^{F S}\right)}{Z_{1}+Z_{2}} \text { and } T_{1}^{F F}=\frac{Z_{1}\left(R_{1}^{F S}-T_{1}^{F S}\right)}{Z_{1}+Z_{2}}
$$

Finally,

$$
\begin{aligned}
R^{F F} & =\frac{Z_{1}^{F}-Z_{2}^{F}}{Z_{1}^{F}+Z_{2}^{F}}+R_{1}^{F F} \frac{1+i}{\sqrt{2}} \sqrt{|\varepsilon|}+\ldots \\
T^{F F} & =1+\frac{Z_{1}^{F}-Z_{2}^{F}}{Z_{1}^{F}+Z_{2}^{F}}+T_{1}^{F F} \frac{1+i}{\sqrt{2}} \sqrt{|\varepsilon|}+\ldots
\end{aligned}
$$

4.2. Slow incident wave. In the Medium 1, for a slow incident wave, one obtains

$$
u_{1}(t, x)=U_{0} e^{i\left(\omega t-k^{1 S} x\right)}+R^{S S} U_{0} e^{i\left(\omega t+k^{1 S} x\right)}+R^{S F} U_{0} e^{i\left(\omega t+k^{1 F} x\right)}
$$

whereas in the Medium 2,

$$
u_{2}(t, x)=T^{S F} U_{0} e^{i\left(\omega t-k^{2 F} x\right)}+T^{S S} U_{0} e^{i\left(\omega t-k^{2 S} x\right)}
$$

Here $R^{S S}, R^{S F}, T^{S F}$, and $T^{S S}$ are the respective reflection and transmission coefficients. Equation (84)-(87) transform into

$$
\begin{gathered}
1+R^{S S}+R^{S F}=T^{S F}+T^{S S} \\
\chi^{1 S}\left(1+R^{S S}\right)+\chi^{1 F} R^{S F}=\chi^{2 F} T^{S F}+\chi^{2 S} T^{S S} \\
-M^{1} k^{1 F}\left(1+\gamma_{K}^{1} \xi^{1 F}\right) R^{S F}+M^{1} k^{1 S}\left(1+\gamma_{K}^{1} \xi^{1 S}\right)\left(1-R^{S S}\right) \\
=M^{2} k^{2 F}\left(1+\gamma_{K}^{2} \xi^{2 F}\right) T^{S F}+M^{2} k^{2 S}\left(1+\gamma_{K}^{2} \xi^{2 S}\right) T^{S S} \\
-M^{1} k^{1 F} \xi^{1 F} R^{S F}+M^{1} k^{1 S} \xi^{1 S}\left(1-R^{S S}\right) \\
=M^{2} k^{2 F} \xi^{2 F} T^{S F}+M^{2} k^{2 S} \xi^{2 S} T^{S S}
\end{gathered}
$$

After dropping the higher-order terms, one gets

$$
\begin{gathered}
1+R^{S S}+R^{S F}=T^{S F}+T^{S S} \\
\varepsilon \chi_{1}^{1 F} R^{S F}+\left(\chi_{0}^{1 S}+\varepsilon \chi_{1}^{1 S}\right)\left(1+R^{S S}\right) \\
=\gamma_{\kappa} \varepsilon \chi_{1}^{2 F} T^{S F}\left(\chi_{0}^{2 S}+\chi_{1}^{2 S} \gamma_{\kappa} \varepsilon\right) T^{S S} \\
-Z_{1} R^{S F}=Z_{2} T^{S F} \\
-\sqrt{\varepsilon} M^{1} k_{0}^{1 F} \xi_{0}^{1 F} R^{S F}+M^{1} k_{0}^{1 S} \xi_{0}^{1 S}\left(1-R^{S S}\right) \\
=\sqrt{\varepsilon} M^{2} k_{0}^{2 F} \xi_{0}^{2 F} T^{S F}+\frac{1}{\sqrt{\gamma_{\kappa}}} M^{2} k_{0}^{2 S} \xi_{0}^{2 S} T^{S S}
\end{gathered}
$$

As in the previous subsection, we seek asymptotic expressions for the transmission and reflection coefficients in the form (96) limiting our analysis 
by the two leading terms only. Putting $\varepsilon=0$ yields:

$$
\begin{aligned}
& R_{0}^{S S}= \frac{-\chi_{0}^{1 S} \frac{1}{\sqrt{\gamma_{\kappa}}} M^{2} k_{0}^{2 S} \xi_{0}^{2 S}+\chi_{0}^{2 S} M^{1} k_{0}^{1 S} \xi_{0}^{1 S}}{\chi_{0}^{1 S} \frac{1}{\sqrt{\gamma_{\kappa}}} M^{2} k_{0}^{2 S} \xi_{0}^{2 S}+\chi_{0}^{2 S} M^{1} k_{0}^{1 S} \xi_{0}^{1 S}} \\
& T_{0}^{S S}=\frac{\chi_{0}^{2 S} \frac{1}{\sqrt{\gamma_{\kappa}}} M^{2} k_{0}^{2 S} \xi_{0}^{2 S}+\chi_{0}^{1 S} M^{1} k_{0}^{1 S} \xi_{0}^{1 S}}{\chi_{0}^{1 S} \frac{1}{\sqrt{\gamma_{\kappa}}} M^{2} k_{0}^{2 S} \xi_{0}^{2 S}+\chi_{0}^{2 S} M^{1} k_{0}^{1 S} \xi_{0}^{1 S}}
\end{aligned}
$$

Hence, using Equations (115) and (117), we obtain

$$
\begin{aligned}
& R_{0}^{S F}=\frac{Z_{2}\left(-1-R_{0}^{S S}+T_{0}^{S S}\right)}{Z_{1}+Z_{2}} \\
& T_{0}^{S F}=\frac{-Z_{1}\left(-1-R_{0}^{S S}+T_{0}^{S S}\right)}{Z_{1}+Z_{2}}
\end{aligned}
$$

A comparison between Equations (97)-(98) and (121)-(122) reveals an asymmetry in the reflection and transmission of the fast and slow waves. A fast incident wave generates a first-order reflected and transmitted slow waves. On the contrary, the slow and the fast waves generated by a slow incident wave are both of the zero order.

\section{ReFleCtion FROM A PERMEABle LAYER}

Consider reflection of a fast incident wave from a permeable layer of thickness $H$. Let this layer, labelled by the superscript ${ }^{2}$, be sandwiched between two media whose properties will be labelled by the superscript ${ }^{1}$, Figure 4 . The classical elasticity predicts tuning effect if the thickness of the layer is equal to one-fourth of the wave length of the signal. For a low-frequency elastic wave, for which the wavelength is much larger than four thicknesses of the layer, the reflections from the top and the bottom interfaces almost cancel each other.

For the Biot's slow and fast waves, however, the picture is different. Both at the top and at the bottom of the layer, an incident wave generates two pairs of transmitted and reflected slow and fast waves. We consider two signals generated by reflection of an incident fast wave from the layer. In the first case, the signal is transmitted into the layer as a slow wave, reflected from the bottom of the layer as a fast wave, and further transmitted back into the upper medium 1 as a fast wave. In the second case, the signal is transmitted into the layer as a fast wave, reflected from the bottom as a slow wave, and transmitted into the upper medium as a fast wave. Figure 4 shows schematically the path of the signal for each of the two configurations. In both cases, the slow wave constitutes only one of the four segments of the whole path. 


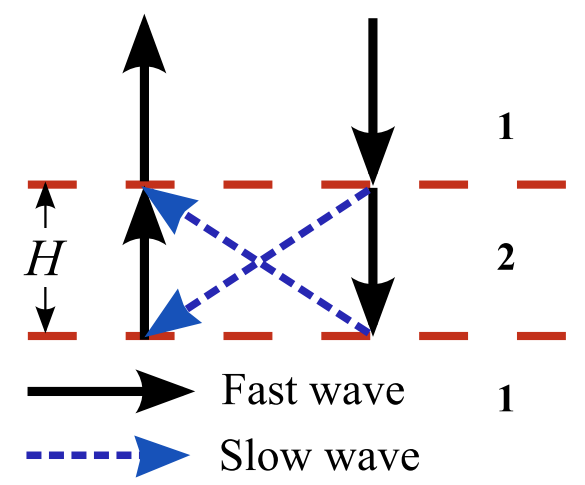

FigURE 4 . Reflection from a permeable layer of type 2 sandwiched between media of type 1 . A fast incident wave generates two coherent reflected fast wave.

Both cases are very similar to each other, so we consider in detail only the first one. Let $U_{0}$ be the amplitude of the incident fast wave. Then, by virtue of Equations (97) and (103), the amplitude of the transmitted slow wave inside the layer is equal to $U_{0}^{1_{f} 2_{s}}=T_{1}^{F S} \sqrt{|\varepsilon|} U_{0}$. The amplitude of the fast wave reflected from the bottom of the layer is equal to

$$
U_{0}^{1_{f} 2_{s} 2_{f}}=R_{0}^{S F} T_{1}^{F S} \sqrt{|\varepsilon|} e^{-a_{S} H} U_{0}
$$

The exponential function in the last equation comes from the slow-wave attenuation. The fast-wave attenuation factor, $a_{F}$, is small of higher order relative to $a_{S}$, see Equation (59). Note that the indices 1 and 2 must be permutated in Equation (121) for a correct evaluation of the reflection coefficient from the bottom $R_{0}^{S F}$. Finally, for the amplitude of the signal reflected from the layer, one obtains the following expression through the amplitude of the original incident wave:

$$
U_{0}^{1_{f} 2_{s} 2_{f} 1_{f}}=T_{0}^{F F} R_{0}^{S F} T_{1}^{F S} \sqrt{|\varepsilon|} e^{-a_{S} H} U_{0}
$$

For a correct evaluation of $T_{0}^{F F}$, the transmission coefficient for the signal reflected from the bottom and crossing the top interface, the indices 1 and 2 must be permutated in Equation (98). Using Equation (61), the $\varepsilon$-dependent factors in the product on the right-hand side of Equation (124) can be gathered in the form:

$$
\psi(|\varepsilon|)=\sqrt{|\varepsilon|} e^{-\frac{\eta}{\kappa} \sqrt{\frac{\gamma_{\beta}+\gamma_{K}^{2}}{2 M \varrho_{f}}} \sqrt{|\varepsilon|} H}
$$

This function attains a maximum value of

$$
\psi_{\max }=\frac{1}{H} \frac{\kappa}{\eta} \sqrt{\frac{2 M \varrho_{f}}{\gamma_{\beta}+\gamma_{K}{ }^{2}}} e^{-1}
$$




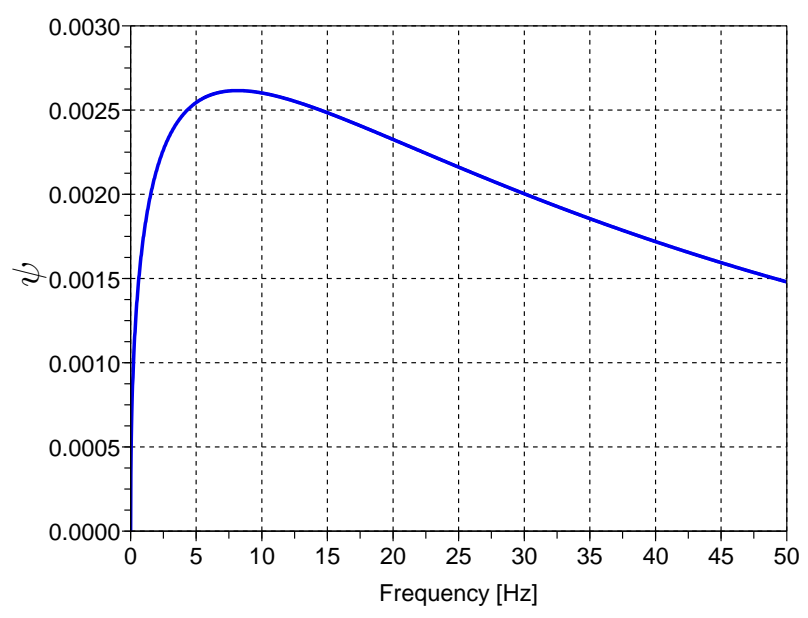

Figure 5. The plot of $\psi$, Equation (125), as a function of frequency. (color online)

at

$$
\sqrt{|\varepsilon|_{\max }}=\frac{1}{H} \frac{\kappa}{\eta} \sqrt{\frac{2 M \varrho_{f}}{\gamma_{\beta}+\gamma_{K}^{2}}}
$$

The peak frequency is

$$
\nu_{\max }=\frac{\kappa}{2 \pi \eta H^{2}} \frac{2 M}{\gamma_{\beta}+\gamma_{K}^{2}}
$$

For example, if $M=10^{+10} \mathrm{~Pa}, \gamma_{\beta}+\gamma_{K}^{2} \approx 2.5, \kappa=1$ Darcy, $\eta=10^{-3}$ Pa-s, and $H=0.5 \mathrm{~m}$, then $\nu_{\max } \approx 8 \mathrm{~Hz}$, Figure 5 . This estimate is in a agreement with field observations of the low-frequency gas shadow [12].

Similarly, for the second path, the reflected signal amplitude is

$$
U_{0}^{1{ }_{f} 2_{f} 2_{s} 1_{f}}=T_{0}^{F F} R_{1}^{F S} T_{0}^{S F} \psi(|\varepsilon|) U_{0}
$$

The transmission coefficients $T_{0}^{F F}$ in Equations (123) and (124) correspond to different direction of wave propagation through the top interface and, in general, are different.

The travel times for both pathes are the same (approximately $30 \mathrm{~ms}$ for the numerical parameters mentioned above), as well as the peak frequencies. The phase shifts due to the travel times also are the same. Therefore, in the superposition of the reflected signals, the amplitudes (124) and (129) sum up. Due to the factor of $\sqrt{|\varepsilon|}$ present in both equations, the absolute value of the reflection coefficient is not large. However, frequently, a reservoir has a layered structure, where the permeability can differ between the layers by orders of magnitude. Summation of the reflections from multiple layer enhances the peak reflection effect with a noticeable time delay relative to the first arrival. 


\section{Summary AND CONCLUSIONS}

Biot's equations of poroelasticity can be obtained from the momentum and mass balance equations. The Hooke's law, adiabatic fluid compressibility, and Darcy flow are the main underlying physical principles. This derivation takes into account the shear stress coming from the spacial variations of the Darcy velocity, which is not a part of the Biot's theory. Additionally, our derivation suggests new physical interpretations for some coefficients of the classical poroelasticity $[4,8]$. For example, the Biot-Willis coefficient $\alpha$ is related to the distribution of the surface of the grains between grain-to-grain and grain-to fluid contact.

Asymptotic analysis of a plane-wave solution at low frequencies leads to explicit relatively simple expressions for the velocity and attenuation of the fast and slow waves. The small parameter is a dimensionless quantity proportional to the product of the fluid mobility, density, and the frequency of the signal. The wave number, velocity, and attenuation factor are expressed as power series with respect to this parameter. The calculations yield that all the coefficients in the power series depend on the mechanical properties of the medium, but neither on the fluid mobility nor on the signal frequency. The requirement that the solution must be physically sensible leads to a coupling relationship between the model parameters.

For the shear-wave solution, the asymptotic-expansion coefficients explicitly depend on the fluid viscosity. Therefore, for a reservoir saturated with a very viscous reservoir fluid, for example, tar, the truncation of the power series after the second leading term may need correction. Such a correction is beyond the scope of the present study.

The obtained asymptotic solutions lead to power-series expressions of the reflection and transmission coefficients for an elastic wave normally crossing a permeable planar interface between two media. It turns out that the leading frequency-dependent term is proportional to the square root of the frequency of the signal. The zero-order terms have been expressed through the acoustic impedances of the media, similarly to the classical theory.

Analysis of the reflection coefficient from a permeable layer (a lens) shows that the reflection of an incident fast wave including one slow-wave segment inside the layer is frequency-dependent and has a peak. The asymptotic relationships make possible an explicit evaluation of the maximum reflection coefficient and the peak-reflection frequency. For a realistic set of parameters, the maximum is attained at a frequency near $10 \mathrm{~Hz}$. Although the amplitude of the reflected signal is proportional to the absolute value of the small parameter and is small, the reflection from a number of such lenses can produce a noticeable effect. Such frequencies have been successfully used for imaging the most permeable regions of a hydrocarbon-bearing reservoir $[21,25]$. 
The obtained asymptotic expressions have suggested useful frequencydependent seismic attributes $[21,25]$. The practical implications of the theory developed here are seismic modeling, inversion, and attribute analysis.

\section{ACKNOWLEDGMENT}

This work has been performed at Lawrence Berkeley National Laboratory (LBNL) of the U.S. Department of Energy (DOE) under Contract No. DE-AC02-05CH11231, University of California at Berkeley, and at the University of Houston. It has been partially supported by DOE grant DE-FC2604NT15503, the UCOil Consortium, and the Reservoir Quantification Laboratory at the University of Houston. The authors are thankful to Dr. Steven

Pride and Dr. Andrea Cortis of LBNL for reviewing the manuscript and for critical remarks and suggestions.

\section{REFERENCES}

[1] M. G. Alishaev and A. K. Mirzadzhanzadeh. On retardation phenomena in filtration theory (in Russian). Neft $i$ Gaz, (6):71-74, 1975.

[2] J. G. Berryman. Elastic wave propagation in fluid-saturated porous media. Journal of Acoustical Society of Amerika, 69:416-424, 1981.

[3] J. G. Berryman and H. F. Wang. Elastic wave propagation and attenuation in a double-porosity dual-permeability medium. Int. J. Rock Mech. and Mining Sci., 37:67-78, 2000.

[4] M. A. Biot. General theory of three dimensional consolidation. Journal of Applied Physics, 12:155-164, 1941.

[5] M. A. Biot. Theory of propagation of elastic waves in a fluid-saturated porous solid. I. Low-frequency range. Journal of the Acoustical Society of America, 28(2):168-178, 1956.

[6] M. A. Biot. Theory of propagation of elastic waves in a fluid-saturated porous solid. II. Higher frequency range. Journal of the Acoustical Society of America, 28(2):179-191, 1956.

[7] M. A. Biot. Mechanics of deformation and acoustic propagation in porous media. Journal of Applied Physics, 33(4):1482-1498, 1962.

[8] M. A. Biot and D. G. Willis. The elastic coefficients of the theory of consolidation. Journal of applied Mechanics, 24:594601, 1957.

[9] B. Budiansky. On the elastic moduli of some heterogeneous materials. J. Mech. Phys. Solids, 13:223-227, 1965.

[10] J. M. Carcione. Viscoelastic effective rheologies for modelling wave propagation in porous media. Geophysical prospecting, 46(3):249-270, 1998.

[11] J. M. Carcione, H. B. Helle, and N. H. Pham. White's model for wave propagation in partially saturated rocks: Comparison with poroelastic numerical experiments. Geophysics, 68:1389-1398, 2003. 
[12] J. P. Castagna, S. Sun, and R. W. Siegfried. Instantaneous spectral analysis: Detection of low-frequency shadows associated with hydrocarbons. The Leading Edge, 22(2):120-127, 2003.

[13] A. Cortis. Dynamic Acoustic Parameters of Porous Media. PhD thesis, Technische Universiteit Delft, Delft, The Netherlans, May 2002.

[14] H. Darcy. Les Fontaines de la ville de Dijon. Victor Dalmont, Paris, 1856.

[15] H. Deresiewicz and J. T. Rice. The effect of boundaries on wave propagation in a liquid-filled porous solid: III. Reflection of plane waves at a free plane boundary (general case). Bulletin of Seismological Society of America, 52(3):595-625, July 1962.

[16] E. Detournay and A. H.-D. Chang. Comprehensive Rock Engineering: Principle, Practice and Projects, Vol II, Analysis and Design Methods, chapter 5. Fundamentals of poroelsticity, pages 113-171. Pergamon Press, 1993.

[17] N. C. Dutta and H. Ode. Seismic reflections from a gas-water contact. Geophysics, 48(02):148-162, 1983.

[18] J. Frenkel. On the theory of seismic and seismoelectric phenomena in a moist soil. Journal of Physics, 8(4):230-241, 1944.

[19] F. Gassmann. Über die Elastizität poröser Medien. Vierteljahrscrift Naturforsch Ges. Zürich, 96:1-23, 1951.

[20] J. Geertsma and D. C. Smit. Some aspects of elastic wave propagation in fluid-saturated porous solids. Geophysics, 26(2):169-181, 1961.

[21] G. Goloshubin, D. Silin, V. Vingalov, G. Takkand, and M. Latfullin. Reservoir permeability from seismic attribute analysis. The Leading Edge, pages 376-381, March 2008.

[22] G. M. Goloshubin and A. V. Bakulin. Seismic reflectivity of a thin porous fluid-saturated layer versus frequency. In 68th SEG Meeting, pages 976-979, New Orleans, 1998.

[23] G. M. Goloshubin, T. M. Daley, and V. A. Korneev. Seismic lowfrequency effects in gas reservoir monitoring VSP data. In SEG Meeting, San Antonio, TX, 2001.

[24] G. M. Goloshubin and V. A. Korneev. Seismic low-frequency effects from fluid-saturated reservoir. In SEG Meeting, Calgary, 2000.

[25] G. M. Goloshubin, V. A. Korneev, D. B. Silin, V. M. Vingalov, and C. VanSchuyver. Reservoir imaging using low frequencies of seismic reflections. The Leading Edge, pages 527-531, May 2006.

[26] G. M. Goloshubin, V. A. Korneev, and V. M. Vingalov. Seismic lowfrequency effects from oil-saturated reservoir zones. In SEG Meeting, Salt Lake City, Utah, 2002.

[27] G. M. Goloshubin and D. B. Silin. Using frequency-dependent seismic attributes in imaging of a fractured reservoir. In SEG Meeting, Houston, TX, 2005. 
[28] B. Gurevich, R. Ciz, and A. I. M. Denneman. Simple expressions for normal incidence reflection coefficients from an interface between fluidsaturated porous materials. Geophysics, 69(6):1372-1377, NovemberDecember 2004.

[29] B. Gurevich, V. B. Zyrianov, and S. L. Lopatnikov. Seismic attenuation in finely layered porous rocks: effects of fluid flow and scattering. Geophysics, 62(1):319-324, 1997.

[30] Z. Hashin. Theory of mechanical behavior of heterogeneous media. Applied Mechanics Reviews, 17:1-9, 1964.

[31] H. B. Helle, N. H. Pham, and J. M. Carcione. Velocity and attenuation in partially saturated rocks: poroelastic numerical experiments. Geophysical Prospecting, 51:551-566, 2003.

[32] M. K. Hubbert. The theory of ground-water motion. Journal of Geology, 48:785-943, 1940.

[33] M. K. Hubbert. Darcy's law and the field equations of the flow of underground fluids. Trans. AIME, 207(7):222-239, 1956.

[34] D. Johnson, J. Koplik, and R. Dashen. Theory of dynamic permeability and tortuosity in fluidsaturated porous media. Journal of Fluid Mechanics, 176:379402, 1987.

[35] S. A. Khristianovich and Y. F. Kovalenko. On elastic drive petroleum reservoir production. Fiz.-Tekhn. Probl. Razrab. Polyezn. Iskop., (1):18-37, 1991.

[36] L. I. Kosachevskii. On the reflection of sound waves from stratified twocomponent media. Applied Mathematics and Mechanics, 25:1608-1617, 1961.

[37] L. D. Landau and E. M. Lifschitz. Fluid mechanics, volume 6 of Series in advanced physics. Addison-Wesley, Reading, MA, 1959.

[38] L. D. Landau and E. M. Lifschitz. Theory of Elasticity. Pergamon Press, Oxford, England, 3 edition, 1986.

[39] M. A. Pelissier, H. Hoeber, N. van der Coevering, and I. F. Jones, editors. Classics of elastic wave theory. Number 24 in Geophysics reprint series. Society of Exploration Geophysicists, Tulsa, OK, 2007.

[40] S. R. Pride. Relationships between seismic and hydrological properties. In Y. Rubin and S. Hubbart, editors, Hydrogeophysics, chapter 9, pages 253-291. Springer, N. Y., 2005.

[41] S. R. Pride and J. G. Berryman. Linear dynamics of double-porosity dual-permeability materials. I. Governing equations and acoustic attenuation. Physical Review E, 68(3):036603:1-10, September 2003.

[42] S. R. Pride and J. G. Berryman. Linear dynamics of double-porosity dual-permeability materials. II. Fluid transport equations. Physical Review E, 68(3):036604:1-10, September 2003.

[43] D. B. Silin, V. A. Korneev, G. M. Goloshubin, and T. W. Patzek. Low-frequency asymptotic analysis of seismic reflection from a fluidsaturated medium. Transport in Porous Media, 62(3):283-305, March 2006. 
[44] J. E. White. Underground sound. Applicatiuon of seismic waves. Elsevier, Amsterdam, 1983.

Lawrence Berkeley National Laboratory, 1 Cyclotron Road, MS 90-1116, Berkeley, CA 94720, USA

E-mail address: DSilin@lbl.gov

University of Houston, 504 Science and Research Bldg 1, Houston, TX 77204

E-mail address: ggoloshubin@uh.edu 